\title{
Superposed epoch analysis of a whistler instability criterion at geosynchronous orbit during geomagnetic storms
}

\author{
E.A. MacDonald ${ }^{\text {a,*}}$, M.H. Denton ${ }^{\text {b }}$, M.F. Thomsen ${ }^{a}$, S.P. Gary ${ }^{a}$ \\ a Los Alamos National Laboratory, P.O. Box 1663, MS D466, Los Alamos, NM 87545, USA \\ ${ }^{\mathrm{b}}$ Department of Communication Systems, Lancaster University, Lancaster, LA1 4WA, UK
}

\section{A R T I C L E I N F O}

\section{Article history:}

Accepted 23 March 2008

Available online 20 April 2008

Keywords:

Magnetic storms and substorms

Plasma waves and instabilities

Plasma sheet

Radiation belts

\begin{abstract}
A B S T R A C T
Enhanced whistler mode waves produced by anisotropic hot plasma-sheet electrons outside the storm-time plasmapause have been suggested as one mechanism for accelerating relativistic outer-belt electrons in the aftermath of geomagnetic storms. Using measurements from the Los Alamos Magnetospheric Plasma Analyzers in geosynchronous orbit, we perform a superposed-epoch study of the storm-time behavior of the inferred plasma-sheet whistler growth parameter. Separate analyses are done for storms that result in strong relativistic electron enhancements and those that do not. The inferred whistler instability is strongest in the midnight-to-dawn sector, where freshly injected plasma-sheet electrons drift into and through the inner magnetosphere. During the main phase of both sets of storms, there is a marked drop in the whistler growth parameter, especially in the prime midnight-to-dawn sector. In the early recovery phase, this parameter is elevated and then returns to more typical values over the next few days. The elevation of the whistler growth parameter persists longer for the electron-enhanced storms than for those that do not produce such enhancements. These results suggest that whistler wave generation is greater during storms yielding enhanced levels of relativistic electrons.
\end{abstract}

(c) 2008 Elsevier Ltd. All rights reserved.

\section{Introduction}

Relativistic electrons in the inner magnetosphere are damaging to spacecraft, and produce high backgrounds in space environment-monitoring instruments (Baker, 2002; MacDonald et al., 2006). Currently, it is not well understood why some storms are more effective than others in producing relativistic electron enhancements a few days after the storm main phase (Reeves et al., 2003). The roles of competing source and loss processes for radiation belt particles are also not resolved (Friedel et al., 2002).

Whistler mode waves are a candidate mechanism for the acceleration of relativistic electrons during geomagnetic storms. Observational and theoretical evidence shows that whistler waves are capable of accelerating

\footnotetext{
* Corresponding author. Tel.: +1505 6060257.

E-mail address: macdonald@lanl.gov (E.A. MacDonald).
}

seed particles to MeV energies (Summers et al., 1998). Observations from the CRRES mission have confirmed earlier results showing that low-latitude whistler wave amplitudes peak around $L=5-7 R_{\mathrm{e}}$ near dawn outside the plasmapause (Burtis and Helliwell, 1969, 1976; Meredith et al., 2001). Of the two whistler chorus bands, the lower one $\left(f<0.5 f_{\text {ce }}\right.$; near the geosynchronous orbit, $\left.\sim 0.5-1.0 \mathrm{kHz}\right)$ is most effective in resonating with and accelerating the energetic electron seed population (Meredith et al., 2003). Whistler waves are associated with increased substorm activity in the recovery phase of geomagnetic storms (Meredith et al., 2001). Akebono wave observations showed that the peak of the whistler intensity propagates to higher L during the recovery phase (Miyoshi et al., 2003, 2007). However, due to the inherent low sampling statistics for in situ wave observations, a global picture of whistler wave activity as a function of geomagnetic storm evolution is difficult to establish. Hence, understanding of the complete relationship 
between enhanced whistler wave activity and enhancements of relativistic electrons remains elusive.

It is widely agreed that enhanced whistler waves can be produced by an electron temperature anisotropy instability, which then scatters the electrons, thereby reducing the anisotropy. The scattering has been shown by simulations and observations to constrain the electron anisotropy to lie at or below the linear theory threshold for the instability, which can be expressed in the form:

$\frac{T_{\perp, \mathrm{e}}}{T_{\mathrm{par}, \mathrm{e}}}-1=\frac{S_{\mathrm{e}}}{\beta_{\mathrm{par}, \mathrm{e}}^{\alpha_{\mathrm{e}}}}$,

with parallel electron beta, $\beta_{\text {par,e }}=8 \pi n_{\mathrm{e}} T_{\text {par,e }} / B_{0}^{2}$ (e.g. Gary and Wang, 1996). $T_{\perp, \mathrm{e}}$ and $T_{\mathrm{par}, \mathrm{e}}$ are the electron temperatures perpendicular and parallel, respectively, to the magnetic field, and $B_{0}$ is the magnetic field magnitude. The parameters $S_{\mathrm{e}}$ and $\alpha_{\mathrm{e}}$ can be derived by fits to linear theory results, assuming a constant maximum growth rate $\gamma_{\text {max }} / \Omega_{\mathrm{e}}$, where $\Omega_{\mathrm{e}}$ is the electron cyclotron frequency (Gary and Wang, 1996). The value of the fitting parameter $\alpha_{\mathrm{e}}$ is generally between 0.4 and 0.6 , but $S_{\mathrm{e}}$ depends more strongly on the growth rate. For a Maxwellian or Kappa distribution, a growth rate of $\gamma / \Omega_{\mathrm{e}}=0.001$ and $\alpha_{\mathrm{e}}=0.6, S_{\mathrm{e}}$ cannot be larger than 0.21 because the waves scatter the thermal electrons so as to reduce the instability growth rate (Gary et al., 2005; Xiao et al., 2006). The whistler instability should therefore impose an upper bound on the electron temperature anisotropy that varies as a function of $\beta_{\text {par,e. The first observational test of this constraint was }}$ presented by Gary et al. (2005) using data from the Cluster 1 spacecraft as it traversed the dayside magnetosheath.

In this paper, we develop a proxy for whistler wave growth based on the properties of the hot plasma-sheet electron population during geomagnetic storms. In this way, the availability of simultaneous plasma particle data from multiple Los Alamos geosynchronous satellites can counteract the relative dearth of plasma wave data in this region. Superposed epoch analysis using electron measurements from the Magnetospheric Plasma Analyzers (MPAs) on multiple Los Alamos geosynchronous satellites (Bame et al., 1993; McComas et al., 1993) is ideal to evaluate the global dynamics of this population as a function of local time and storm phase. For this study, these comparisons are made for sets of storms with and without a strong relativistic electron response at geosynchronous orbit in order to elucidate the role of whistler waves in the mechanisms of relativistic electron enhancement and dynamics.

\section{Data}

Two sets of storms are selected following the criteria of O’Brien et al. (2001, 2003), also applied by Smith et al. (2004b) in their study of chorus waves using ground observations. We take the same event list as Smith et al. (2004b), expanded to include storms in the 3 earlier years which are covered by the geosynchronous particle database. There are also some slight differences in our list compared to that used by Smith et al. (2004b) due to sporadic data gaps from either source. Selected storms have a minimum Dst less than $-50 \mathrm{nT}$ and cover the time period 1989-2002. Of this set only isolated storms are chosen, specifically those without storm minima closer than 4 days. Following the example of O'Brien et al. (2003) and Smith et al. (2004b), storms are sorted into events and non-events based on whether or not the "noonreconstructed" $1.8-3.5 \mathrm{MeV}$ electron flux measured by Los Alamos National Laboratory geosynchronous satellites 48-72 $\mathrm{h}$ after the Dst minimum exceeds $0.5 \mathrm{e}^{-} / \mathrm{cm}^{2} \mathrm{~s}$ sr keV. As described by O'Brien et al. (2001), the noon-reconstructed flux is based on a statistical technique to remove the local time variations in radiation belt particles and normalize the fluxes to that of a virtual geosynchronous spacecraft located at noon. In summary, our study includes 138 event storms (more enhanced post-storm $\mathrm{MeV}$ electron response) and 183 non-event storms (less enhanced $\mathrm{MeV}$ electron response).

Event selection based on these criteria, specifically only the post-storm fluxes, is justifiable since the prestorm flux was shown to be uncorrelated with the poststorm flux by Reeves et al. (2003). As shown in Fig. 1 (and also by Smith et al., 2004b) both sets of storms have very similar superposed epoch Dst profiles, although events have longer elevated $K_{\mathrm{p}}$ levels. This is consistent with the findings of O'Brien et al. (2001) that the relativistic electron response is uncorrelated with the size of the storm (based on Dst) but is related to the strength of convection (as proxied by $K_{\mathrm{p}}$ ) and recovery phase substorm activity.

The extensive database of LANL-GEO MPA plasma spectrometer measurements from seven geosynchronous satellites is used in the present superposed epoch analysis The electron moments are calculated for the population from $30 \mathrm{eV}$ to $45 \mathrm{keV}$ according to the methods described in Thomsen et al. (1999). Superposed epoch analysis of the plasma moments and other parameters, with zero epoch time at the minimum of Dst, was done following the method of Denton et al. (2005), used also by Denton et al. (2006) and Lavraud et al. (2005). Parallel electron beta was calculated at each point using the dynamic T89 model (Tysganenko, 1989), since in situ measurements of magnetic field strength were not available. Each analysis was done separately for the event and non-event sets of storms.

Fig. 2(a-d) shows the electron density and temperature as functions of local time and epoch time for the sets of events and non-events. Notably, for these parameters the storm-time characteristics of the events and non-events are very similar to the sets of co-rotating interaction region (CIR) and coronal mass ejection-driven storms, respectively, as studied by Denton et al. (2006). The average density of the electron population peaks postmidnight near Dst minimum and reaches a minimum near noon. This is consistent with the plasmasheet moving inward of geosynchronous orbit in the main phase of the storm, observed first near midnight and then spreading in local time (Denton et al., 2006). Non-events have higher density than events around Dst minimum and in the storm recovery phase.

The temperature (defined as one-third the sum of twice the perpendicular temperature and the parallel 
a

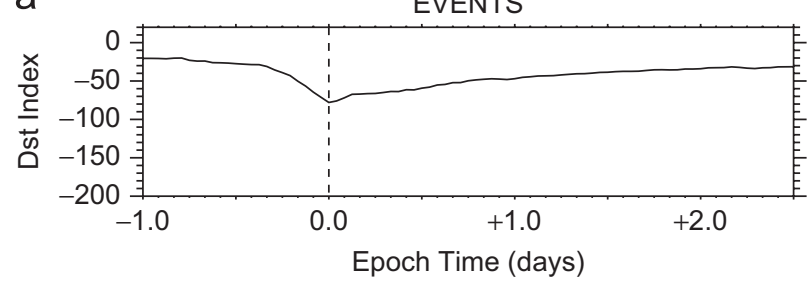

C

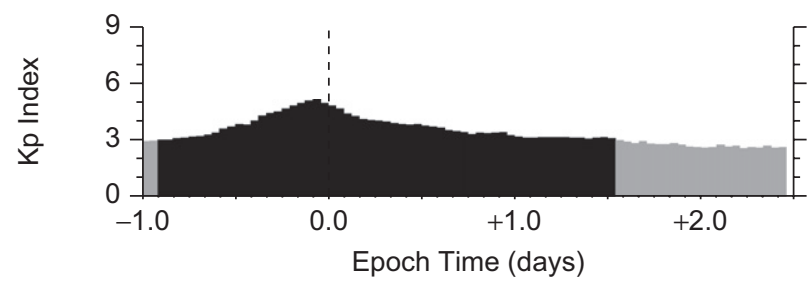

b

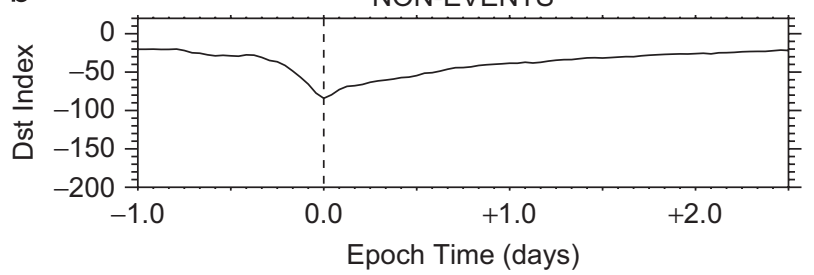

d

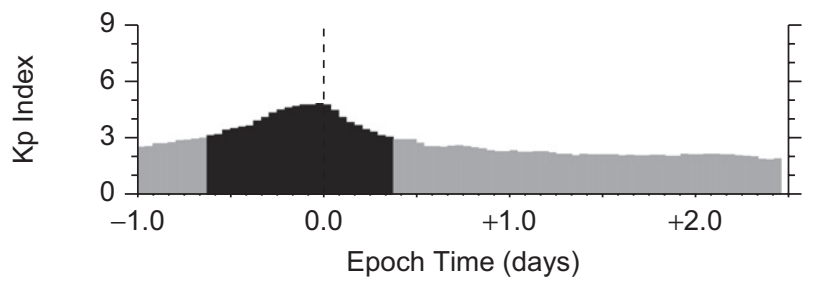

Fig. 1. Superposed epoch analysis of Dst and Kp indices for (a) events with high relativistic electron response, and (b) non-events without such response. The epoch time for each storm is the time of minimum Dst.

temperature) for the events is enhanced more at Dst minimum and remains a factor of nearly two higher than for non-events in the midnight region during the storm recovery phase.

Fig. 2(e and $\mathrm{f}$ ) shows the electron temperature anisotropy, $A_{\mathrm{e}}$, which is calculated as the ratio of the perpendicular temperature to the parallel temperature. As discussed by Denton et al. (2005), $A_{\mathrm{e}}$ is closest to unity at midnight due to the isotropy of freshly arriving tail plasma. It then increases towards noon as particles drift through the inner magnetosphere and precipitate (Korth et al., 1999). The most distinctive feature of the epochtime behavior is the wide local-time range of the decrease in $A_{\mathrm{e}}$ around the storm main phase. The region of local time and epoch time where this occurs is consistent with the enhanced electron densities observed in Fig. 2( $a$ and b) being a quite isotropic plasmasheet population. This occurs for both event and non-event storms, and was noted earlier by Denton et al. (2006). An alternate explanation for the observed isotropy may be that an abundance of waves rapidly isotropizes the distributions.

As shown in Fig. 3(a and b) the values and evolution of the parallel plasma beta look fairly similar for the events and the non-events because the aforementioned differences in the density and temperature (particularly in the recovery phase) counteract each other. However, events have a higher beta in the recovery phase, while nonevents have a higher beta in the storm main phase, around the time of Dst minimum.

In Eq. (1), $S_{\mathrm{e}}$ and $\alpha_{\mathrm{e}}$ are parameters determined by fits to the theoretical whistler instability threshold conditions. Here we use our geosynchronous data set to calculate the observational analog of $S_{\mathrm{e}}$, what we call the whistler growth parameter

$\sum_{\mathrm{e}}=\beta_{\mathrm{par}, \mathrm{e}}^{\alpha_{\mathrm{e}}}\left(\frac{T_{\perp, \mathrm{e}}}{T_{\mathrm{par}, \mathrm{e}}}-1\right)$,

and we follow the theoretical results of Gary et al. (2005) in choosing $\alpha_{e}=0.60$. Results are plotted in Fig. 3(c and d).
Relatively large values of $\Sigma_{\mathrm{e}}$ are interpreted as corresponding to strongly unstable conditions, whereas smaller values of this parameter represent weakly unstable or stable conditions.

The superposed epoch results show enhanced values of $\Sigma_{\mathrm{e}}$ between midnight and noon, in general agreement with the global distribution of chorus amplitude inferred from in situ wave measurements (Meredith et al., 2001). Our measure of whistler-mode wave growth seems to correspond to the equatorial lower-band chorus Meredith et al. (2001) observed during active times. There is a marked decrease in $\Sigma_{\mathrm{e}}$ in the $\sim 12 \mathrm{~h}$ prior to Dst minimum (i.e. in the storm main phase) from midnight to dawn (see Section 3 for more detail). Values for the events appear slightly higher than for the non-events in the recovery phase. Since an order of magnitude smaller whistler growth rate only changes $S_{\mathrm{e}}$ from 0.21 to 0.15 , the modest difference in the observed values of $\Sigma_{\mathrm{e}}$ between events and non-events is significant (Gary et al., 2005).

\section{Discussion}

The average values of $\Sigma_{\mathrm{e}}$ for both sets of storms shown in Fig. 3 are lower than the value of 0.21 anticipated for a modest growth rate of $0.001 \Omega_{\mathrm{e}}$, based on a Maxwellian electron velocity distribution. Since the superposed epoch values for $\Sigma_{\mathrm{e}}$ are significantly below the value for marginal stability of the whistler mode, we examine the variability of the points that were averaged within each hourly bin of local time vs. epoch time. Fig. 4 shows the results of a whistler anisotropy threshold test similar to Gary et al. (2005). For a whistler instability threshold condition corresponding to a growth rate of $0.001 \Omega_{\mathrm{e}}, S_{\mathrm{e}}=0.21$ and $\alpha_{\mathrm{e}}=0.6$; then Eq. (1) yields the straight line shown on the $\log$ plots of $\left(A_{\mathrm{e}}-1\right)$ versus $\beta_{\text {par,e }}$ (where the slope depends on $\alpha_{\mathrm{e}}$ and the intercept on $S_{\mathrm{e}}$ ). The proximity to the threshold instability line of measured values of anisotropy at a given beta indicates approaching the limit of stability. In Fig. 4, all the individual measurements of $\left(A_{\mathrm{e}}-1\right)$ and 

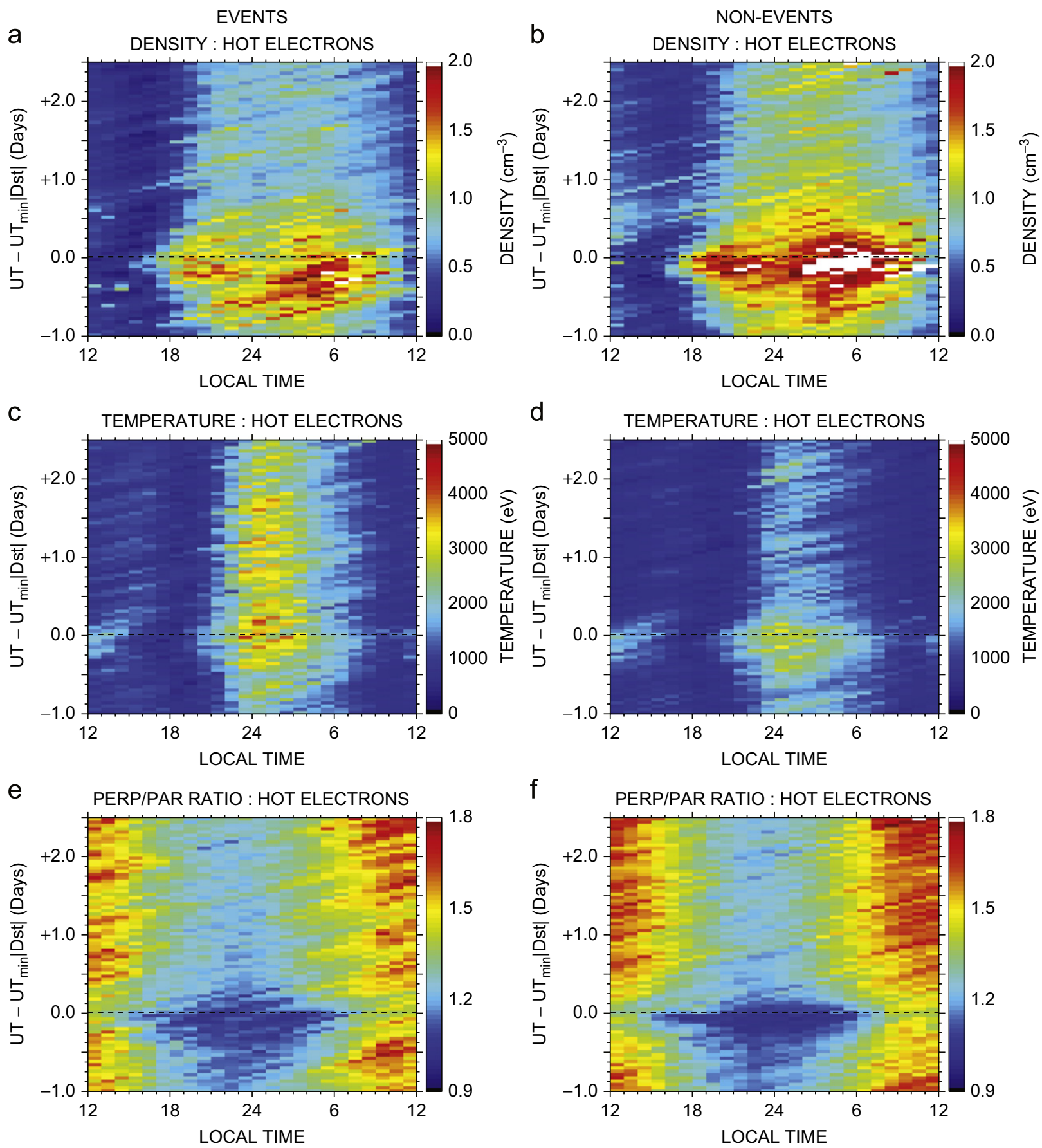

Fig. 2. Superposed epoch analysis of hot electron parameters for events with high relativistic electron response (left column) and non-events without such response (right column). Epoch time covers 3.5 days with zero epoch at Dst minimum. Panels (a) and (b) show the density, (c) and (d) the temperature, and (e) and (f) the temperature anisotropy for the hot electron population.

$\beta_{\text {par,e }}$ for event storms are plotted for the full range of epoch time at each hour of local time, according to the color-coding described in plot. We see that the points (especially from midnight to dawn) approach the instability threshold line, though this was not evident in the superposed epoch plots. Wave-induced particle scattering is a fast, bursty process acting to decrease the electron anisotropy, and thereby reduce the instability growth rate. Thus it is not surprising that only some of the points approach this limit and that the average value (in the superposed epoch plots) lies well below the instability threshold criterion. Fig. 4 confirms that whistler waves are 


\section{EVENTS}

a
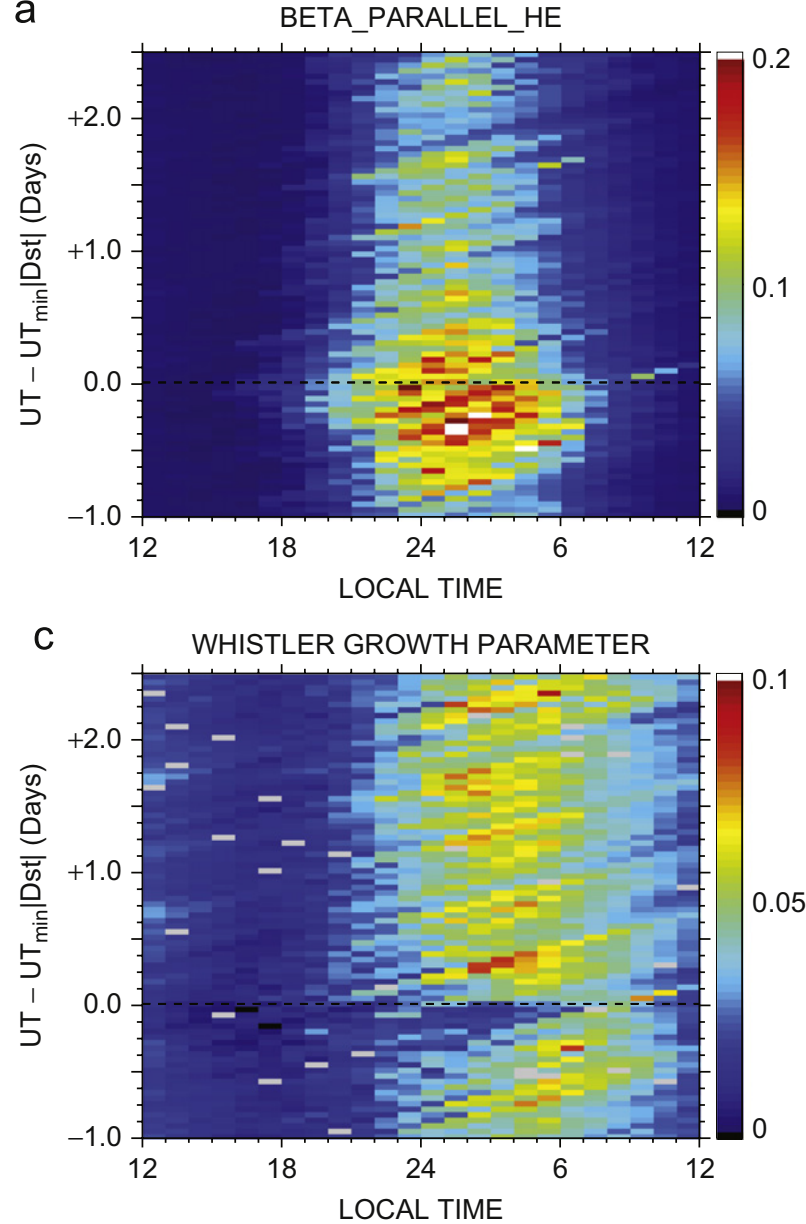

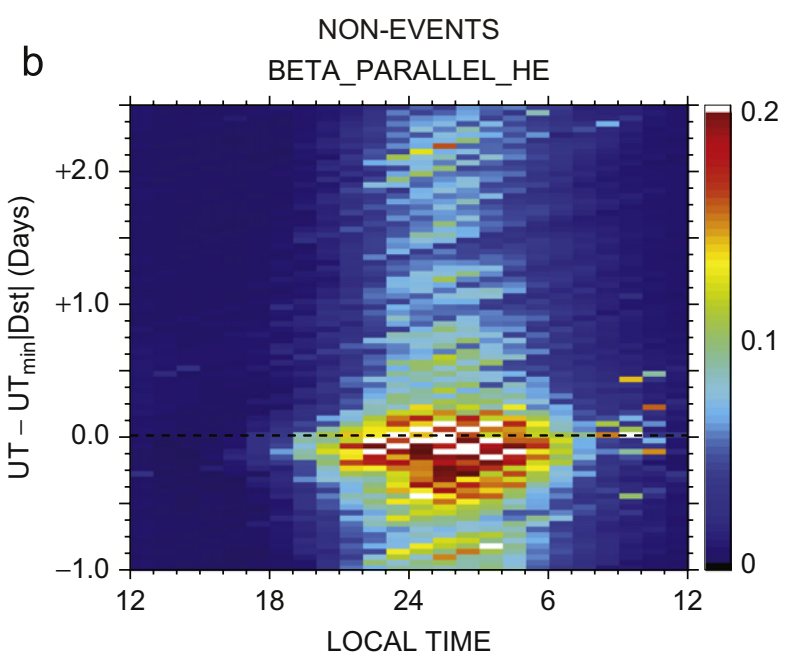

d

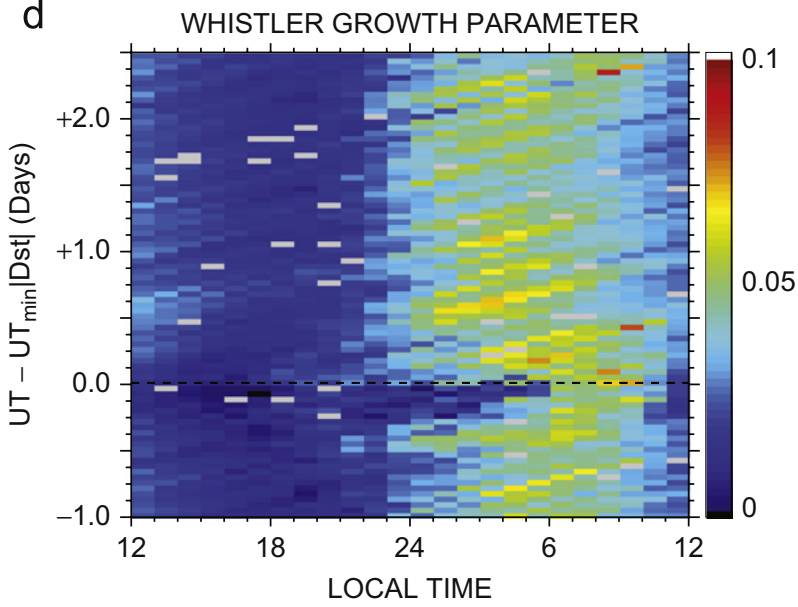

Fig. 3. (Same format as Fig. 2.) Panels (a) and (b) show the electron beta, and (c) and (d) the whistler growth parameter for the hot electron population as derived in the text.

acting, and their local-time distribution is consistent with the average distribution of lower band equatorial chorus described by Meredith et al. (2001) based on CRRES data. We extend that result since CRRES coverage near geosynchronous orbit was limited. The difference in stability between post-midnight (a) and near dusk (c) is most striking. Only event storms are shown in this example as not much difference is evident when comparing events to non-events using this representation.

Fig. 5(a) shows the epoch time behavior of the whistler growth parameter averaged over local time from midnight to noon for both events and non-events. Plotted in this manner, there is a more distinct increase in $\Sigma_{\mathrm{e}}$ observed in the events than the non-events during the late storm recovery phase from $\sim 1$ day after Dst minimum to $\sim 4$ days. At other times, the distributions are quite similar. Fig. 5 also shows that the values for $\Sigma_{\mathrm{e}}$ recede to quiet-time behavior approximately 4-5 days after the minimum of Dst and that the average value is quite variable.

Fig. 5(b) shows the distribution of lower band chorus waves measured by a ground-based VLF receiver from
Halley station $(L=4.3)$, Antarctica, for the set of storms studied by Smith et al. (2004b) (adapted from Fig. 2, Smith et al., 2004b). The similarity of Fig. 5(a) and (b) is quite striking. This confirms that our technique to derive the whistler growth parameter from electron measurements accurately corresponds to direct wave measurements. For events and non-events, Fig. 5(b) shows an approximately two-fold decrease in the magnetic field spectral density at whistler chorus frequencies during the main phase. Smith et al. (2004a, b) attributed this decrease to an ionospheric attenuation effect during the storm main phase. Since this decrease is also evident in the geosynchronous growth parameter, it may in fact be a product of reduced growth in the magnetosphere, rather than a propagation effect. During the recovery phase, Fig. 5(b) shows that chorus intensities increased by a factor of $\sim 3$ (relative to the average quiet-time value). Very similar to our space-based inference of the whistler wave distribution, the groundbased observations showed more whistler waves for events than non-events, with the difference maximizing $\sim 1-2$ days after Dst minimum is reached. Further study of 

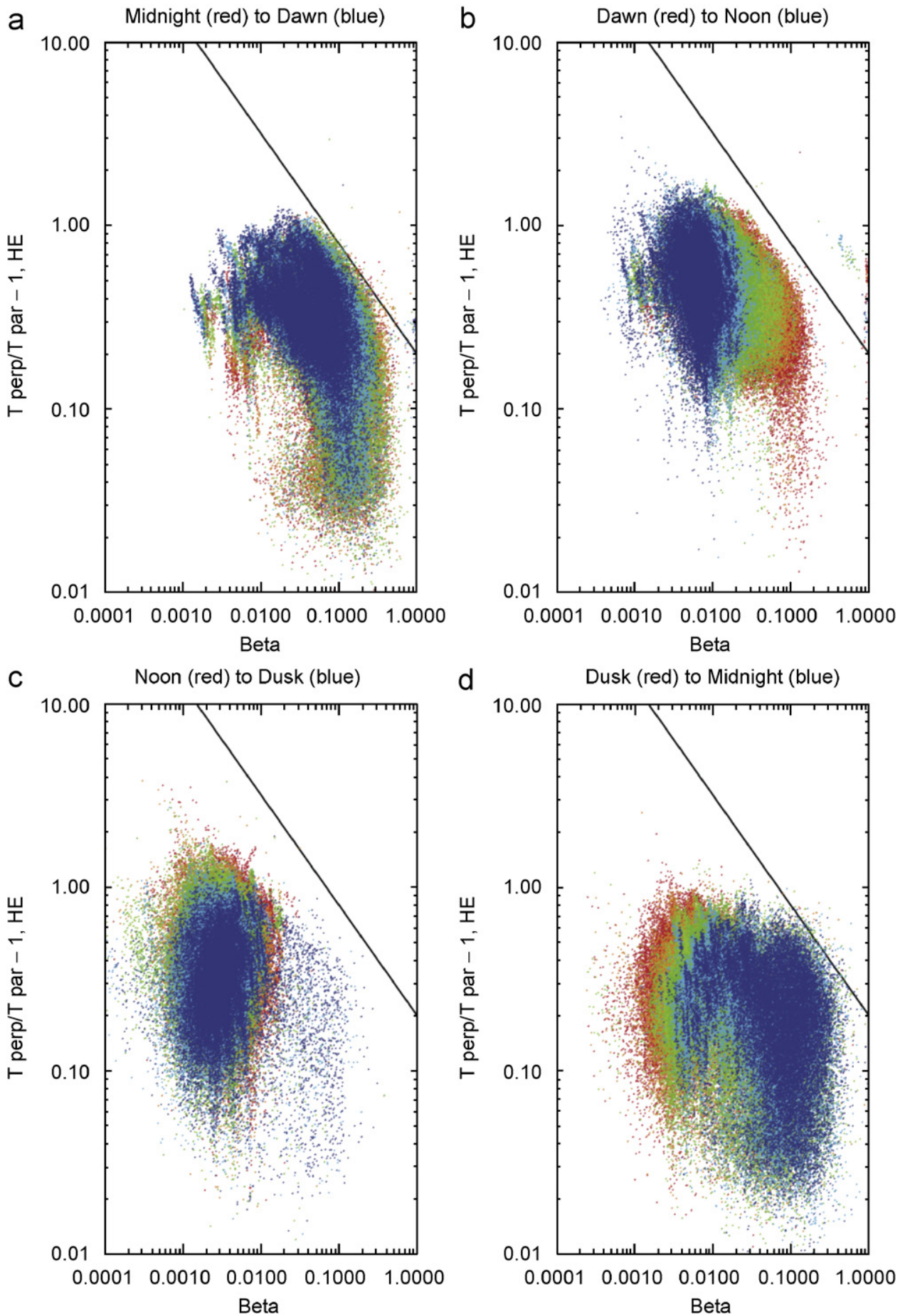

Fig. 4. The results of the whistler instability test described in the text performed for data from -1 to +3 days after Dst minimum for the event set of storms. Each color represents all the measurements of $A-1$ vs. Beta for $1 \mathrm{~h}$ of local time. Each panel represents $6 \mathrm{~h}$ of local time with the color spanning red to blue as hour increases. The solid line shows the theoretical prediction of whistler instability.

the similarities between Fig. 5(a) and (b) is warranted, but beyond the scope of this paper.

Using in situ observations, Miyoshi et al. $(2003,2007)$ also show evidence that the occurrence of observable unstable whistler waves may decrease at geosynchronous orbit during the main phase of geomagnetic storms because the chorus is most active first at lower $L$, and the activity subsequently propagates outward. By contrast, Meredith et al. (2002) report observations of plentiful whistler waves during the storm main phase. 


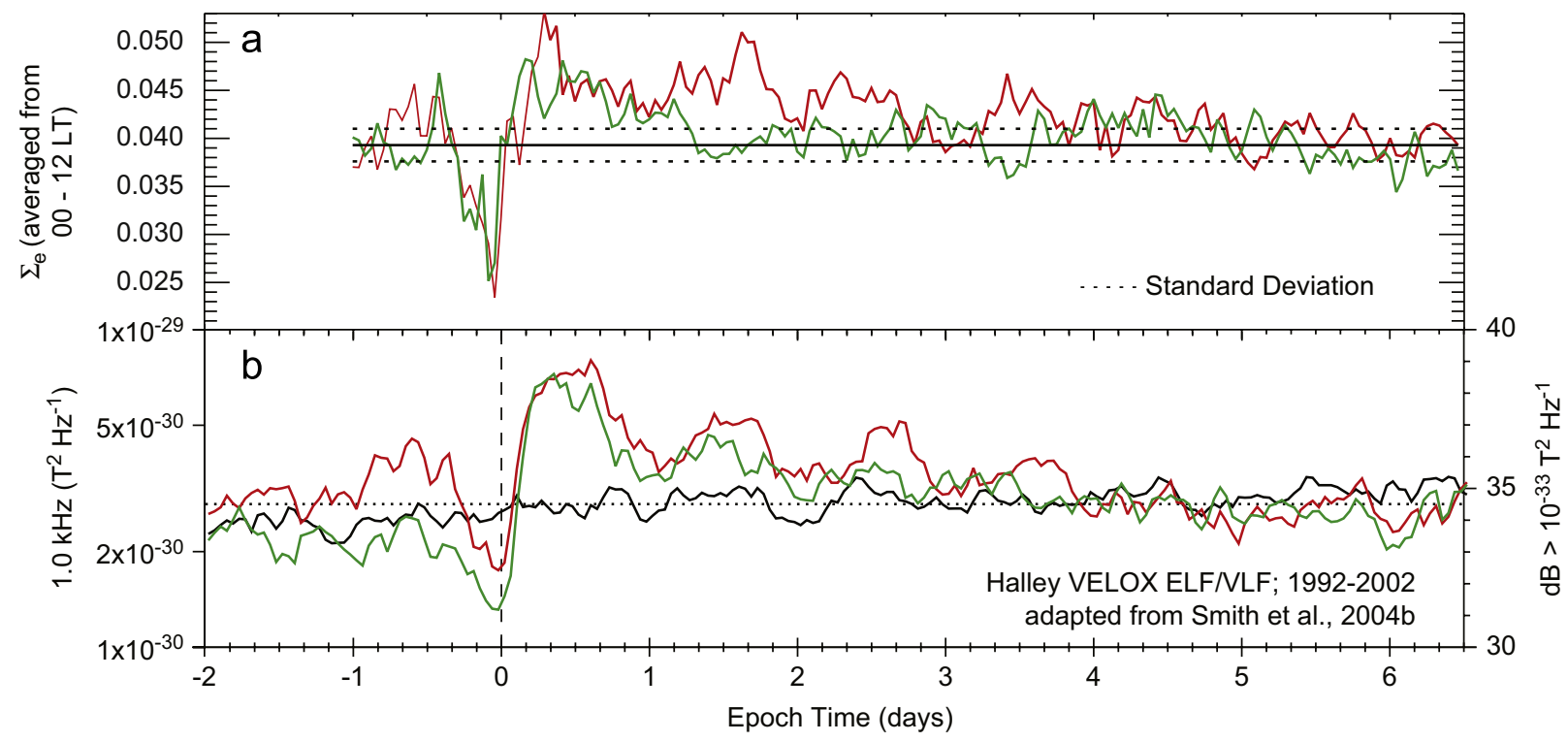

Fig. 5. (a) Average $\Sigma_{\mathrm{e}}$ from midnight to noon local time versus epoch day (starting 1 day before Dst minimum). Events are shown in red and non-events in green. The average quiet-time value is shown in black and the standard deviation about the average by the dashed lines. The values of $\Sigma_{\mathrm{e}}$ return to normal after $\sim 4$ days of storm recovery. After Smith et al. (2004b), panel (b) shows the magnetic field spectral density of ground-observed lower band chorus waves for a superposed epoch analysis of similarly defined events (red) and non-events (green). Also shown is the result for randomized epoch times (black). (Reprinted with permission from A. J. Smith and the American Geophysical Union.)

The significant differences in local time, L shell, and latitudinal coverage between our observations at geosynchronous orbit and previous measurements by the CRRES and Akebono satellites may account for some of these discrepancies in the observations of chorus during the storm main phase.

\section{Conclusions}

A superposed epoch study of electron parameters measured at geosynchronous orbit relating to the growth of the whistler anisotropy instability was performed on magnetic storms with and without significant post-storm relativistic electron enhancements. Overall, the Dst profile of the two sets of storms looked very similar, although events with enhancements showed higher $K_{\mathrm{p}}$ in the recovery phase, consistent with the supposition that higher solar wind speed storms (e.g. CIR events) are correlated with enhanced relativistic electron production in the magnetosphere (O'Brien et al., 2001). The electron plasma population was overall less dense and hotter from Dst minimum through the recovery phase for events compared with non-events. During the storm main phase, a dense, isotropic electron population arrived in the midnight region and became more anisotropic towards noon, reflecting drift paths and precipitation through the inner magnetosphere. For events relative to non-events, the electron parallel beta was significantly higher during the recovery phase though lower during the storm main phase.

The observed whistler growth parameter, defined by Eq. (2), exhibits the same limiting behavior expected by quasi-linear theory and demonstrated previously for the magnetosheath and the solar wind (Gary and Wang, 1996; Gary et al., 2005; Stverak et al., 2008). This parameter was enhanced for the events compared with the non-events, particularly in the late recovery phase. In addition, the temporal behavior of whistler wave growth during storms was very similar to that derived by Smith et al. (2004b) using ground-based wave observations.

In conclusion, this evidence suggests that whistler wave generation is higher for storms which result in enhanced relativistic electron production. The time with the strongest enhancement in the whistler growth parameter is $\sim 1.5$ days after storm maximum, which is also the time significant for the production and observation of relativistic electrons. This suggests that whistler wave growth as inferred from the source electron population at geosynchronous orbit can indeed be observed and related to relativistic electron enhancements. However, since theory suggests that the electron acceleration should be most effective right outside the plasmapause (Summers et al., 1998), the difference may be much larger at smaller L. In situ particle measurements could be used to examine the strength and timing predicted for whistler wave growth closer to the plasmapause during geomagnetic storms.

This instability analysis technique using particle measurements as a proxy is useful because direct wave measurements are not always available. The whistler growth parameters could be examined for specification or re-analysis studies over a full solar cycle (e.g. O'Brien and Lemon, 2007). This technique could also be applied to other regions and other wave modes. 


\section{Acknowledgments}

We thank Benoit Lavraud, Paul O'Brien, Andy Smith, Athina Varotsou, Reiner Friedel, and Geoff Reeves for helpful input and discussions. We also thank the World Data Centre at Rutherford Appleton Laboratory for providing the Dst and $\mathrm{Kp}$ index data. This work was conducted at Los Alamos National Laboratory under the auspices of the Department of Energy, with partial support from internal R\&D funding for the DREAM project and the NASA LWS program.

\section{References}

Baker, D., 2002. How to cope with Space Weather. Science 297, 1486.

Bame, S.J., McComas, D.J., Thomsen, M.F., Barraclough, B.L., Elphic, R.C. Glore, J.P., Chavez, E.P., Evans, J.C., Wymer, F.J., 1993. Magnetospheric plasma analyzer for spacecraft with constrained resources. Review of Scientific Instruments 64, 1026-1033.

Burtis, W.J., Helliwell, R.A., 1969. Banded chorus-a new type of VLF radiation observed in the magnetosphere by OGO 1 and OGO 3 . Journal of Geophysical Research 74, 3002.

Burtis, W.J., Helliwell, R.A., 1976. Magnetospheric chorus: occurrence patterns and normalized frequency. Planetary and Space Science 24 (11), 1007-1023.

Denton, M.H., Thomsen, M.F., Korth, H., Lynch, S., Zhang, J.C., Liemohn, M.W., 2005. Bulk plasma properties at geosynchronous orbit. Journal of Geophysical Research 110, A07223.

Denton, M.H., Borovsky, J.E., Skoug, R.M., Thomsen, M.F., Lavraud, B., Henderson, M.G., McPherron, R.L., Zhang, J.C., Liemohn, M.W., 2006. Geomagnetic storms driven by ICME- and CIR-dominated solar wind. Journal of Geophysical Research 110, A07S07.

Friedel, R.H.W., Reeves, G.D., Obara, T., 2002. Relativistic electron dynamics in the inner magnetosphere-a review. Journal of Atmospheric and Solar Terrestrial Physics 64, 265.

Gary, S.P., Wang, J.J., 1996. Whistler anisotropy instability: electron anisotropy upper bound. Journal of Geophysical Research 101, 10749.

Gary, S.P., Lavraud, B., Thomsen, M.F., Lefebvre, B., Schwartz, S., 2005. Electron anisotropy constraint in the magnetosheath: cluster observations. Geophysical Research Letters 32, L13109.

Korth, H., Thomsen, M.F., Borovsky, J.E., McComas, D.J., 1999. Plasma sheet access to geosynchronous orbit. Journal of Geophysical Research 104, 25047-25061.

Lavraud, B., Denton, M.H., Thomsen, M.F., Borovsky, J.E., Friedel, R.H., 2005. Superposed epoch analysis of cold, dense plasma sheet access to geosynchronous orbit. Annales Geophysicae 23, 1-11.

MacDonald, E., Thomsen, M., Funsten, H., 2006. Background in channel electron multiplier detectors due to penetrating radiation in space. IEEE Transactions on Nuclear Science 53, 3.

McComas, D.J., Bame, S.J., Barraclough, B.L., Donart, J.R., Elphic, R.C., Gosling, J.T., Moldwin, M.B., Moore, K.R., Thomsen, M.F., 1993. Magnetospheric plasma analyzer: initial 3-spacecraft observations from geosynchronous orbit. Journal of Geophysical Research 98 13453-13465.

Meredith, N.P., Horne, R.B., Anderson, R.R., 2001. Substorm dependence of chorus amplitudes: implications for the acceleration of electrons to relativistic energies. Journal of Geophysical Research 106, 13,165 .

Meredith, N.P., Horne, R.B., Iles, R.H.A., Thorne, R.M., Heynderickx, D., Anderson, R.R., 2002. Outer zone relativistic electron acceleration associated with substorm-enhanced whistler mode chorus. Journal of Geophysical Research 107, 1144.

Meredith, N.P., Horne, R.B., Thorne, R.M., Anderson, R.R., 2003. Favored regions for chorus-driven electron acceleration to relativistic energies in the Earth's outer radiation belt. Geophysical Research Letters 30 (16), 1871.

Miyoshi, Y., Morioka, A., Obara, T., Misawa, H., Nagai, T., Kasahara, Y., 2003. Rebuilding process of the outer radiation belt during the November 3,1993, magnetic storm: NOAA and EXOS-D observations. Journal of Geophysical Research 108, 1004.

Miyoshi, Y., Morioka, A., Kataoka, R., Kasahara, Y., Mukai, T., 2007. Evolution of the outer radiation belt during the November 1993 storms driven by corotating interaction regions. Journal of Geophysical Research 112, A05210.

O’Brien, T.P., Lemon, C.L., 2007. Reanalysis of plasma measurements at geosynchronous orbit. Space Weather 5, S03007.

O’Brien, T.P., McPherron, R.L., Sornette, D., Reeves, G.D., Friedel, R., Singer, H.J., 2001. Which magnetic storms produce relativistic electrons at geosynchronous orbit? Journal of Geophysical Research 106, 15,533.

O’Brien, T.P., Lorentzen, K.R., Mann, I.R., Meredith, N.P., Blake, J.B., Fennel, J.F., Looper, M.D., Milling, D.K., Anderson, R.R., 2003. Energization of relativistic electrons in the presence of ULF wave power and MeV microbursts: evidence for dual ULF and VLF acceleration. Journal of Geophysical Research 108, 1329.

Reeves, G.D., McAdams, K.L., Friedel, R.H.W., O’Brien, T.P., 2003. Acceleration and loss of relativistic electrons during geomagnetic storms. Geophysical Research Letters 30, 10.

Smith, A.J., Horne, R.B., Meredith, N.P., 2004a. Ground observations of chorus following geomagnetic storms. Journal of Geophysical Research 109, A02205.

Smith, A.J., Meredith, N.P., O'Brien, T.P., 2004b. Differences in groundobserved chorus in geomagnetic storms with and without enhanced relativistic electron fluxes. Journal of Geophysical Research 109, A11204.

Stverak, S., Travnicek, P., Maksimovic, M., Marsch, E., Fazakerley, A.N. Scime, E.E., 2008. Electron temperature anisotropy constraints in the solar wind. Journal of Geophysical Research 113, A03103.

Summers, D., Thorne, R.M., Xiao, F., 1998. Relativistic theory of waveparticle resonant diffusion with application to electron acceleration in the magnetosphere. Journal of Geophysical Research 103, 20,487.

Thomsen, M.F., Noveroske, E., Borovsky, J.E., McComas, D.J., 1999. Calculation of Moments from Measurements by the Los Alamos Magnetospheric Plasma Analyzer. LA Rep. LA-13566-MS. Los Alamos Nat. Lab., Los Alamos, NM 87545.

Tysganenko, M., 1989. A magnetospheric magnetic field model with a wrapped tail current sheet. Planetary and Space Science 37, 5-20.

Xiao, F., Zhou, Q., Zheng, H., Wang, S., 2006. Whistler instability threshold condition of energetic electrons by kappa distribution in space plasmas. Journal of Geophysical Research 111, A08208. 